Rev. Int. Contam. Ambie. 33 (1) 109-116, 2017

DOI: 10.20937/RICA.2017.33.01.10

\title{
PRODUCCIÓN DE BIOGÁS MEDIANTE CODIGESTIÓN ANAEROBIA DE EXCRETAS DE BORREGO Y RUMEN ADICIONADAS CON LODOS PROCEDENTES DE UNA PLANTA DE AGUAS RESIDUALES
}

\author{
Marco Antonio SARABIA MÉNDEZ, José Ramón LAINES CANEPA*, José Aurelio SOSA OLIVIER y \\ Erika ESCALANTE ESPINOSA
}

División Académica de Ciencias Biológicas, Universidad Juárez Autónoma de Tabasco. Carretera VillahermosaCárdenas, kilómetro 0.5, Entronque Bosques de Saloya, Villahermosa Centro, Tabasco, México, C. P. 86150

*Autor para correspondencia: josra_2001@yahoo.com.mx

(Recibido noviembre 2015; aceptado junio 2016)

Palabras clave: biodigestor, excreta, metano, residuos

\section{RESUMEN}

Hoy en día se necesitan nuevas fuentes de producción de energía. Además, hay un incremento de los residuos agrícolas que representan un problema ambiental. El propósito del presente estudio fue comparar la digestión anaerobia de excretas de borrego con la codigestión anaerobia de excretas de borrego más lodos de aguas residuales y la codigestión anaerobia de excretas de borrego más rumen. Los resultados mostraron que el tratamiento T1 (excretas de borrego-lodos de aguas residuales), produjo una menor cantidad de biogás, 35.47 L, comparado con el tratamiento T2 (excretas de borregorumen) y el tratamiento T3 (excretas de borrego), con $42.14 \mathrm{~L}$ y $43.83 \mathrm{~L}$ respectivamente. El análisis estadístico de la composición de biogás mostró que los valores de $\mathrm{CH}_{4}$ y CO , no fueron significativamente diferentes $(\mathrm{P}=0.5660$ y 0.2589$)$. Los valores de $\mathrm{H}_{2} \mathrm{~S}(\mathrm{P}=0.0379)$, mostraron que la concentración fue menor en el tratamiento $\mathrm{T} 1$ que en el tratamiento T3. Si los residuos agrícolas son utilizados apropiadamente con codigestión anaerobia pueden ser convertidos en energía.

Key words: biodigestor, manure, methane, waste

\begin{abstract}
Nowadays, new energy production sources are needed. Also, there is an increase in agricultural waste, which represents an environmental problem. The purpose of the present study was to compare the anaerobic digestion of sheep manure feces with the anaerobic co-digestion of sheep feces + wastewater sludge, and with the anaerobic co-digestion of sheep feces + rumen. The results showed that the treatment T1 (sheep feces + wastewater sludge) produced a lower quantity of biogas, $35.47 \mathrm{~L}$, compared to the treatment T2 (sheep feces + rumen) and treatment T3 (sheep feces only), with 42.14 $\mathrm{L}$ and $43.83 \mathrm{~L}$, respectively. Statistical analysis of the biogas composition showed that the values of $\mathrm{CH}_{4}$ and $\mathrm{CO}_{2}$ were not significantly different $(\mathrm{P}=0.5660$ and 0.2589$)$. The values for $\mathrm{H}_{2} \mathrm{~S}(\mathrm{P}=0.0379)$, showed that the concentration was less in treatment $\mathrm{T} 1$ than in treatment T3. If these agricultural wastes are used appropriately with codigestion, they can be converted into energy.
\end{abstract}




\section{INTRODUCCIÓN}

La digestión anaerobia es una tecnología para el tratamiento de residuos orgánicos que no sólo permite dar solución a su manejo, sino que también permite reducir las emisiones de gases de efecto invernadero, aprovechar (mediante la producción de biogás) el potencial energético de los residuos y obtener un producto rico en nutrientes, útil como fertilizante. En el proceso de codigestión anaerobia se combinan varias mezclas de sustratos orgánicos biodegradables, logrando aumentar el potencial de producción de biogás por kilogramo de mezcla degradado. Existe un gran número de ejemplos donde se ha demostrado la efectividad de este proceso. Borowski y Weatherley (2013) reportaron que la adición de $30 \%$ de estiércol sólido de aves de corral a lodos de una planta de tratamiento de aguas incrementó la producción total de gas en un $50 \%$. Por otra parte, la codigestión de estiércol de aves con el lodo dio lugar a una mayor eficiencia de eliminación de solidos volátiles totales (SVT) de $43.16 \%$ a $49.35 \%$, en comparación con la digestión utilizando sólo lodos (33.85\% a $36.33 \%$ ). Borowski et al. (2013) encontraron que, en la codigestión de lodos de una planta de tratamiento de aguas residuales con excremento de cerdo y aves, con un $30 \%$ (en peso) de estiércol de cerdo, aumenta en $40 \%$ la producción de biogás, pero con el uso de estiércol de aves (10\% en peso), la producción de biogás disminuyó, debido a la inhibición de la metanogénesis, por presencia de amoniaco libre. Las excretas de borrego son de los residuos menos estudiados en cuanto al proceso de digestión anaerobia. Karki et al. (2005) reportan un contenido de SVT de $74.89 \%$ en base seca para excretas de borrego. Álvarez y Liden (2009) obtuvieron biogás con una concentración de $49.9 \%$ de metano, utilizando una mezcla con $12.6 \%$ de SVT de excretas de borrego y $87.4 \%$ de agua. Así mismo obtuvieron una reducción de $19.54 \%$ de SVT. Ashekuzzaman y Tjalfe (2011) obtuvieron biogás a partir de excretas de borrego con un contenido de metano de $53 \%$ y una reducción de SVT de $26 \%$. Dentro de la problemática del excremento de ganado se encuentra el riesgo de enfermedades para la población humana por el consumo de agua contaminada con: 1) estiércol conteniendo bacterias patógenas; la más común es Escherichia coli que causa diarrea y gases abdominales (LeJeune y Wetzel 2007), 2) contenidos altos de nitratos que reducen la capacidad de transporte de oxígeno en la sangre, conocida como metahemoglobinemia (Miner et al. 2000), 3) presencia de hormonas, principalmente estrógenos, relacionadas con una reducción en la cantidad de esperma en humanos (Sharpe y Skakkebaek 1993), 4) además del impacto ambiental como generación de gases de efecto invernadero, eutrofización de cuerpos de agua y sobrecarga de nutrientes en suelos. En los últimos años el incremento en la producción de ganado ovino en México ha sido mayor que el de otro tipo. Según el Servicio de Información Agroalimentaria y Pesquera (SIAP) (2012a) el número de cabezas en el 2003 era de 6819771 y en el 2012 fue de 8405902 , mientras que para ganado bovino pasó de 31476600 a 31925181 del 2003 al 2012. Esta tendencia se observa en los diversos tipos de explotación de ovinos en el estado de Tabasco, la cual ha tenido un impulso importante. Según la SIAP (2012b), en la entidad se estimó un hato ovino de 75110175 cabezas que, comparado con las 57660 cabezas del 2003 representa un incremento en la producción del $30.37 \%$, esto conlleva a un aumento en las excretas producidas por este tipo de ganado y, por ende, a los problemas que estas excretas ocasionan.

Por lo anterior, el objetivo del presente trabajo fue evaluar la codigestión de excretas de borrego con lodos de una planta de tratamiento de aguas residuales comparado con el uso de excretas de borrego con contenido gástrico ruminal vacuno (en adelante rumen), logrando un tratamiento adecuado de los mismos que no solo permita eliminar los problemas que ocasionan, sino además aprovechar su potencial energético mediante la producción de biogás. Se busca beneficiar a las personas que se dedican a la crianza de borrego y proporcionar una alternativa económicamente viable para el tratamiento de estos residuos con la producción de biogás. Asimismo, contar con una fuente de energía renovable y lograr beneficios ambientales al disminuir la cantidad de residuos orgánicos y las emisiones de gases de efecto invernadero (GEI).

\section{MATERIALES Y MÉTODOS}

\section{Obtención del sustrato y cosustrato}

Las excretas de borrego (sustrato) fueron obtenidas del rancho "El Rodeo", en el municipio de Jalapa, Tabasco. El rumen (cosustrato) fue obtenido del rastro municipal del municipio de Cunduacán, Tabasco, en ambos casos se utilizó el método de cuarteo para obtener una muestra compuesta representativa, de acuerdo con la NMX-AA-015-1985 (SE 1985). Los lodos (cosustrato) se obtuvieron de una planta de tratamiento de aguas residuales del municipio de Cunduacán, Tabasco. 


\section{Caracterización del sustrato y cosustrato}

Se realizaron las determinaciones de porcentaje de humedad, sólidos volátiles totales y cenizas, con base en las normas NMXAA161984 (SE 1984a), NMXAA-034-SCFI-2001 (SE 2001) y NMX-AA-18-1984 (SE 1984b), respectivamente. Los sólidos totales (ST) se calcularon por diferencia de $100 \%$ menos el porcentaje de humedad (Bux et al. 2012).

\section{Sistema de codigestión}

Para las unidades experimentales se utilizaron botellas de reacción de $1 \mathrm{~L}$ acopladas mediante una manguera a una bolsa para biogás de $1 \mathrm{~L}$ que sirvió como reservorio. Las botellas contenían $800 \mathrm{~g}$ de mezcla, la cual constaba de $10 \%$ de SV $(90 \%$ pertenecían al sustrato y el $10 \%$ al cosustrato). Se realizaron 3 tratamientos: T1) excretas de borregolodos, T2) excretas de borrego-rumen y T3) excretas de borrego. Cada tratamiento se realizó por triplicado. Las unidades experimentales estuvieron dentro de una estufa a $27^{\circ} \mathrm{C}$ para simular la temperatura promedio del estado de Tabasco, mismas que se mantuvieron en agitación diariamente a $900 \mathrm{rpm}$ durante $20 \mathrm{~min}$ (Fig. 1).

\section{Monitoreo del proceso}

Parámetros físicos y químicos. Como el proceso es un sistema anaerobio, se construyeron sistemas con las mismas condiciones a las unidades experimentales en tubos de reacción de $20 \mathrm{~mL}$, para realizar mediciones semanales. Los parámetros analizados fueron: oxígeno disuelto (OD), $\mathrm{pH}$ y potencial de óxido reducción (POR), durante las 14 semanas que duró el proceso. Es importante resaltar que las mediciones iniciales y finales se llevaron a cabo directamente en las unidades experimentales.

Nutrientes. Se analizó el contenido de nutrientes al inicio y al final del proceso, directamente en las unidades experimentales mediante el uso de un medidor multiparámetro marca Hanna ${ }^{\circledR} 83225$ con precisión de $\pm 10 \mathrm{mg} / \mathrm{L}$ para nitrógeno, $\pm 5 \mathrm{mg} / \mathrm{L}$ para fósforo $\mathrm{y} \pm 30 \mathrm{mg} / \mathrm{L}$ para potasio.

Sólidos volátiles totales. Se determinó la remoción de SVT midiéndolos al inicio y al final del proceso, con base en la NMX-AA-034-SCFI-2001 (SE 2001).

Caracterización del biogás. Cada unidad experimental tenía una bolsa para biogás. Una vez llena, se analizaba el contenido, con un equipo Dragër® modelo X-am 7000.

\section{Análisis estadístico}

Se llevó a cabo un análisis de varianza de un factor, para encontrar diferencias significativas de los tres tratamientos evaluados (T1, T2 y T3), en la producción de $\mathrm{CH}_{4}, \mathrm{CO}_{2}$ y $\mathrm{H}_{2} \mathrm{~S}$. Asimismo, se aplicó una prueba múltiple de Tukey para determinar diferencias entre cada tratamiento. Para ello, se utilizó el programa STATGRAPHICS ${ }^{\circledR}$ Centurion XV.II.

\section{Diseño experimental}

Se utilizó un diseño completamente aleatorizado de un factor. Cada unidad experimental consistía de una botella de reacción de $1 \mathrm{~L}$. El número de observaciones en cada tratamiento se realizó por triplicado: tres unidades experimentales contenían excretas de borrego-lodos, tres unidades excretas de borregorumen y tres unidades más, excretas de borrego.

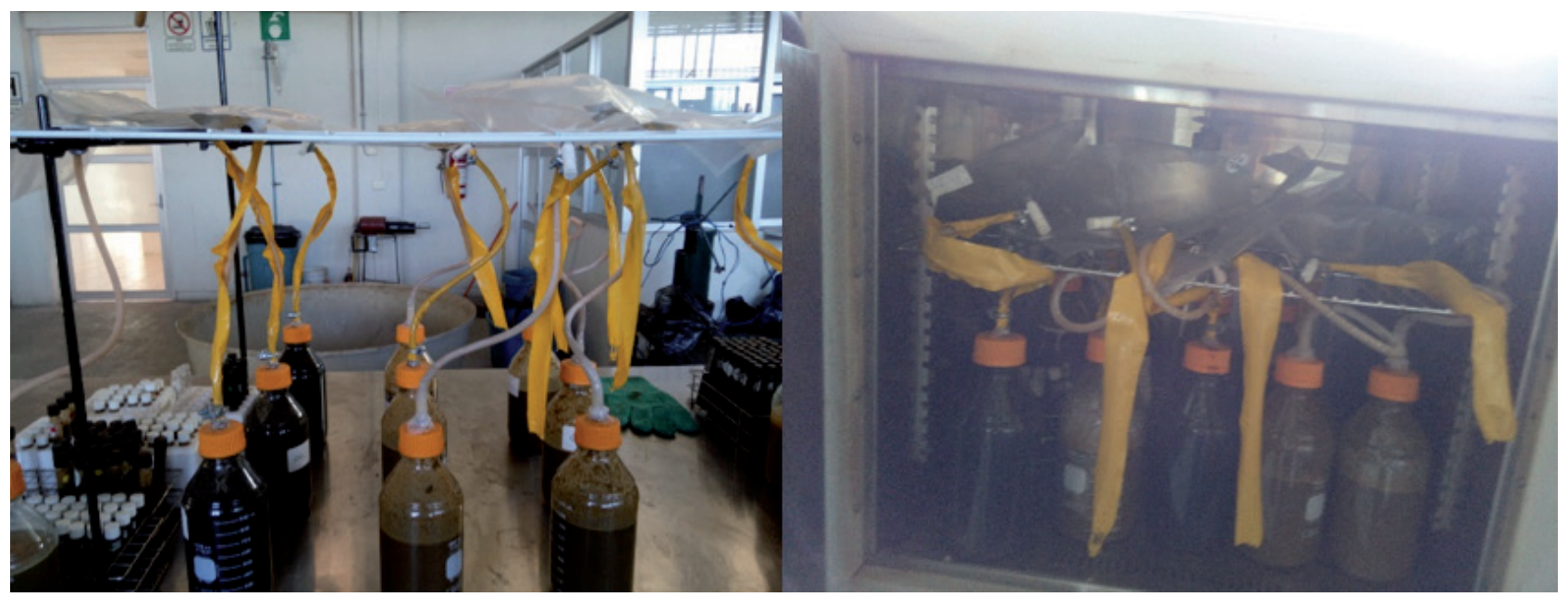

Fig. 1. Izquierda: sistema de codigestión montado. Derecha: unidades experimentales a temperatura constante $\left(27^{\circ} \mathrm{C}\right)$ 


\section{RESULTADOS Y DISCUSIÓN}

\section{Caracterización de los residuos}

El contenido de humedad para el rumen fue 1.6 veces mayor que el de las excretas de borrego y 1.2 veces mayor que para los lodos (Cuadro I). En cuanto a los SVT, el lodo presenta menor cantidad, mientras las excretas de borrego y el rumen tienen cantidades parecidas. Los lodos tienen más de $60 \%$ de cenizas en base seca, mientras los otros dos residuos alrededor de $10 \%$, es decir, los lodos contienen una mayor cantidad de minerales que los otros residuos. Esta característica puede permitir una mayor velocidad de crecimiento de las bacterias durante el proceso, lo cual se traduce en una producción de biogás con mayor rapidez.

CUADRO I. CARACTERÍSTICAS DE LOS RESIDUOS EVALUADOS

\begin{tabular}{lcccc}
\hline Residuo & $\begin{array}{c}\text { Humedad } \\
(\%)\end{array}$ & $\begin{array}{c}\text { SVT* } \\
(\%)\end{array}$ & $\begin{array}{c}\text { Cenizas* } \\
(\%)\end{array}$ & $\begin{array}{c}\text { ST } \\
(\%)\end{array}$ \\
\hline Excretas de borrego & 49.35 & 82.12 & 12.51 & 50.65 \\
Rumen & 82.08 & 80.21 & 10.36 & 17.92 \\
Lodos & 69.76 & 10.96 & 65.81 & 30.24 \\
\hline
\end{tabular}

$\mathrm{SVT}=$ sólidos volátiles totales, $\mathrm{ST}=$ sólidos totales, $*$ base seca

\section{Parámetros físicos y químicos pH}

En la figura 2, se muestra la variación del pH para cada tratamiento. El T1 y T3, muestran un $\mathrm{pH}$ inicial cercano a 7.5, mientras el T2 tiene un $\mathrm{pH}$ inicial superior a 8 . A partir de la semana 1 , el $\mathrm{pH}$ de los 3 tratamientos se mantuvo en un valor cercano a 6.5. En las primeras 3 semanas se observan los pH más bajos (con excepción de la semana 9 para el caso de las excretas de borrego que presentan el

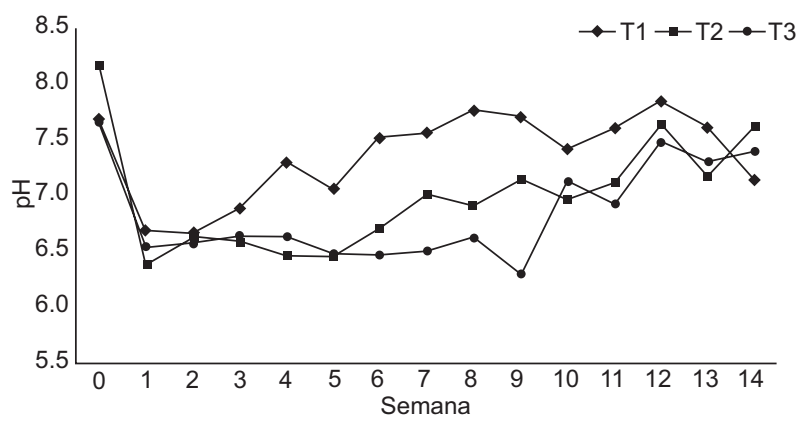

Fig. 2. Variación del pH durante la codigestión. T1 = excretas de borrego-lodos, T2 = excretas de borrego-rumen, $\mathrm{T} 3=$ excretas de borrego
pH más bajo durante el proceso), lo que indica que este periodo corresponde a la etapa acidogénica. Al finalizar las 14 semanas de proceso, los tres tratamientos terminaron con un $\mathrm{pH}$ similar, cercano a 7.5. De acuerdo con Lay et al. (1997), el rango óptimo de $\mathrm{pH}$ para el proceso de digestión anaerobia es de 6.6 a 7.6, por lo que el $\mathrm{pH}$ se mantuvo en condiciones óptimas para los tres tratamientos durante el proceso.

\section{Potencial de óxido reducción (POR)}

En la figura 3 se observa que desde el inicio de proceso tuvo un POR favorable, ya que para los tres tratamientos fue disminuyendo durante la primera semana y posteriormente comenzó a incrementarse a partir del día 36. De acuerdo con Flotats et al. (2001) el POR óptimo para el proceso es por debajo de $-300 \mathrm{mV}$, valor que se mantuvo durante los primeros 36 días para el T1 y T3, y durante los primeros 29 para el T2. Durante el resto del proceso los valores oscilaron entre $-200 \mathrm{mV}$ y $-300 \mathrm{mV}$, aunque los valores obtenidos no fueron los mejores en las condiciones óptimas, estos son aceptables.

\section{Oxígeno disuelto (OD)}

La figura 4, muestra los valores de $\mathrm{OD}$, observándose que el comportamiento fue parecido para los tres tratamientos, aumentando durante las primeras 4 semanas y disminuyendo después de éstas.

\section{Nutrientes}

El cuadro II presenta el contenido de nitrógeno en el biodigestato, observando que el nitrógeno final es mucho menor que el inicial. Los T1 y T3 finalizaron con un porcentaje de nitrógeno en masa con valores similares, mientras el T2 contuvo menos nitrógeno. En el cuadro III se observa la cantidad del nutriente

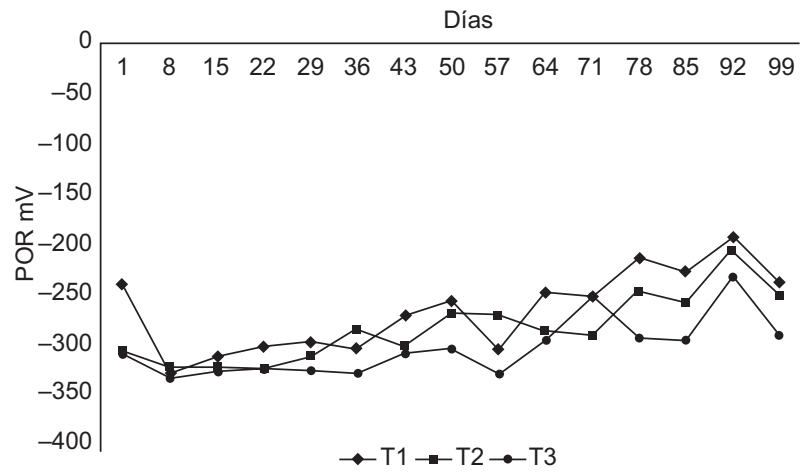

Fig. 3. Comportamiento del potencial de óxido reducción (POR) durante la codigestión. T1 = excretas de borrego-lodos, $\mathrm{T} 2=$ excretas de borrego-rumen, $\mathrm{T} 3=$ excretas de borrego 


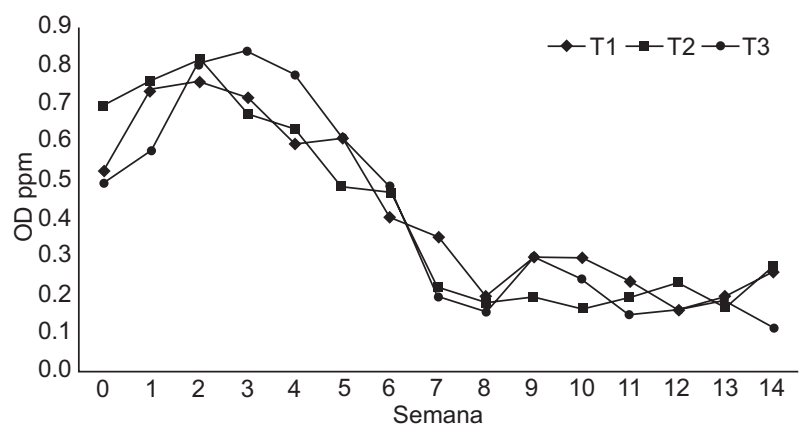

Fig. 4. Comportamiento del oxígeno disuelto (OD) durante la codigestión. T1 = excretas de borrego-lodos, $\mathrm{T} 2=$ excretas de borrego-rumen, $\mathrm{T} 3=$ excretas de borrego

fósforo al iniciar y finalizar el proceso, nuevamente el T2 es el que contiene menos de este nutriente. El cuadro IV presenta la cantidad de potasio al iniciar y finalizar el proceso, el T3 presentó casi el doble de este nutriente con respecto a los otros dos tratamientos.

\section{Sólidos volátiles totales (SVT)}

El cuadro V, muestra el porcentaje de SVT removidos en cada tratamiento. El T3 tiene el mayor porcentaje de remoción. La cantidad de biogás obtenida por g de SV es menor para el T1 y similar para los T2 y T3. La remoción de SVT (incluso para el T3 que sólo es excreta de borrego) es mayor a lo reportado por Álvarez y Liden (2009) y por Ashekuzzaman y Tjalfe (2011) quienes obtuvieron una reducción de $19.54 \%$ y $26 \%$ de SV, respectivamente, para excretas de borrego. En el caso del T1, se obtuvo una mayor remoción de SV que lo reportado por Borowski y Weatherley (2013), 33.85-36.33\% de SVT para lodos solamente. De acuerdo con los resultados del análisis de varianza, no existen diferencias significativas en la remoción de SVT, para los tres tratamientos evaluados.

\section{Cuantificación y caracterización del biogás}

El cuadro $\mathbf{V}$, presenta la cantidad de biogás que se obtiene porcada tratamiento, asícomo la caracterización

CUADRO II. CONTENIDO DE NITRÓGENO COMO $\mathrm{NO}_{3}{ }^{-}$

\begin{tabular}{lcccc}
\hline Tratamiento & $\begin{array}{c}\text { Nitrógeno inicial } \\
(\mathrm{mg} / \mathrm{L})\end{array}$ & $\begin{array}{c}\text { Nitrógeno Semana } 6 \\
(\mathrm{mg} / \mathrm{L})\end{array}$ & $\begin{array}{c}\text { Nitrógeno final } \\
(\mathrm{mg} / \mathrm{L})\end{array}$ & $\begin{array}{c}\text { Nitrato final } \\
(\%)\end{array}$ \\
\hline T1 & $33333 \pm 3055$ & $5833 \pm 1443$ & $15000 \pm 0$ & 1.28 \\
$\mathrm{~T} 2$ & $26000 \pm 5292$ & $14167 \pm 7638$ & $6667 \pm 7127$ & 0.59 \\
$\mathrm{~T} 3$ & $48667 \pm 10263$ & $3750 \pm 750$ & $14500 \pm 1803$ & 1.28 \\
\hline
\end{tabular}

$\mathrm{T} 1=$ excretas de borrego-lodos, $\mathrm{T} 2=$ excretas de borrego-rumen, $\mathrm{T} 3=$ excretas de borrego, \pm desviación estándar

CUADRO III. CONTENIDO DE FÓSFORO COMO $\mathrm{P}_{2} \mathrm{O}_{5}$

\begin{tabular}{lcccc}
\hline Tratamiento & $\begin{array}{c}\text { Fósforo inicial } \\
(\mathrm{mg} / \mathrm{L})\end{array}$ & $\begin{array}{c}\text { Fósforo Semana } 6 \\
(\mathrm{mg} / \mathrm{L})\end{array}$ & $\begin{array}{c}\text { Fósforo final } \\
(\mathrm{mg} / \mathrm{L})\end{array}$ & $\begin{array}{c}\text { Fósforo final } \\
(\%)\end{array}$ \\
\hline T1 & $115333 \pm 27277$ & $38833 \pm 24497$ & $66000 \pm 72794$ & 5.61 \\
T2 & $114667 \pm 18583$ & $14000 \pm 1323$ & $39333 \pm 31943$ & 3.45 \\
T3 & $100000 \pm 106000$ & $21250 \pm 21583$ & $60000 \pm 51333$ & 5.31 \\
\hline
\end{tabular}

$\mathrm{T} 1$ = excretas de borrego-lodos, T2 = excretas de borrego-rumen, T3 = excretas de borrego, \pm desviación estándar

CUADRO IV. CONTENIDO DE POTASIO COMO $\mathrm{K}_{2} \mathrm{O}$

\begin{tabular}{lcccc}
\hline Tratamiento & $\begin{array}{c}\text { Potasio inicial } \\
(\mathrm{mg} / \mathrm{L})\end{array}$ & $\begin{array}{c}\text { Potasio Semana } 6 \\
(\mathrm{mg} / \mathrm{L})\end{array}$ & $\begin{array}{c}\text { Potasio final } \\
(\mathrm{mg} / \mathrm{L})\end{array}$ & $\begin{array}{c}\text { Potasio final } \\
(\%)\end{array}$ \\
\hline T1 & $53333 \pm 15275$ & $62500 \pm 41307$ & $55833 \pm 22684$ & 4.73 \\
$\mathrm{~T} 2$ & $44667 \pm 13013$ & $37167 \pm 7006$ & $56667 \pm 25042$ & 4.97 \\
T3 & $60000 \pm 4000$ & $34250 \pm 3382$ & $93333 \pm 105633$ & 8.26 \\
\hline
\end{tabular}

$\mathrm{T} 1$ = excretas de borrego-lodos, T2 = excretas de borrego-rumen, $\mathrm{T} 3$ = excretas de borrego, \pm desviación estándar 
CUADRO V. CUANTIFICACIÓN Y CARACTERIZACIÓN DEL BIOGÁS

\begin{tabular}{lcccccc}
\hline Tratamiento & Biogás & $\begin{array}{c}\text { SVT removidos } \\
(\%)\end{array}$ & $\begin{array}{c}\mathrm{CH}_{4} \\
(\%)\end{array}$ & $\begin{array}{c}\mathrm{CO}_{2} \\
(\%)\end{array}$ & $\begin{array}{c}\mathrm{O}_{2} \\
(\%)\end{array}$ & $\begin{array}{c}\mathrm{H}_{2} \mathrm{~S} \\
(\mathrm{ppm})\end{array}$ \\
\hline $\mathrm{T} 1$ & $35.47 \pm 11.36$ & $50.43 \pm 16.15$ & 64.22 & 30.61 & 5.55 & 91.63 \\
$\mathrm{~T} 2$ & $42.14 \pm 1.26$ & $61.47 \pm 1.80$ & 64.22 & 31.67 & 4.11 & 134.9 \\
$\mathrm{~T} 3$ & $43.84 \pm 1.13$ & $64.71 \pm 1.60$ & 61.12 & 33.07 & 5.81 & 133.95 \\
\hline
\end{tabular}

$\mathrm{T} 1=$ excretas de borrego-lodos, $\mathrm{T} 2$ = excretas de borrego-rumen, $\mathrm{T} 3=$ excretas de borrego, \pm desviación estándar

del mismo. E1 T3 es el que produce mayor cantidad de biogás. La composición del gas obtenido tuvo un mayor porcentaje de metano $\left(\mathrm{CH}_{4}\right)$ en los T1 y T2 comparados con el T3. También se observó que el porcentaje de $\mathrm{CH}_{4}$ fue mayor al de $\mathrm{CO}_{2}$, concordando con los resultados obtenidos de forma teórica. De acuerdo con Weiland (2010), esto sucede porque una parte del $\mathrm{CO}_{2}$ se encuentra solubilizado en el biodigestato. El análisis de varianza de un factor, muestra que no existen diferencias significativas (P $=0.5660)$ entre los tres tratamientos evaluados en la producción de metano con un $95 \%$ de confianza. Sin embargo, se observa una tendencia del T1 y el T2 (excretas de borrego-lodo y excretas de borregorumen) hacia presentar valores porcentuales mayores (Fig. 5). En cuanto a la producción porcentual de bióxido de carbono, el análisis de varianza de un factor, muestra que no existen diferencias estadísticas significativas $(\mathrm{P}=0.2589)$ entre los tres tratamientos evaluados. No obstante, se observa una tendencia del T3 (excretas de borrego) hacia presentar valores porcentuales mayores (Fig. 6). Las concentraciones de ácido sulfhídrico muestran que existen diferencias estadísticas significativas $(\mathrm{P}=0.0379)$ entre los tres tratamientos evaluados (Fig. 7). Se observa que el T3 (excretas de borrego) genera la concentración más baja, seguida del T2 (excretas de borrego-rumen).

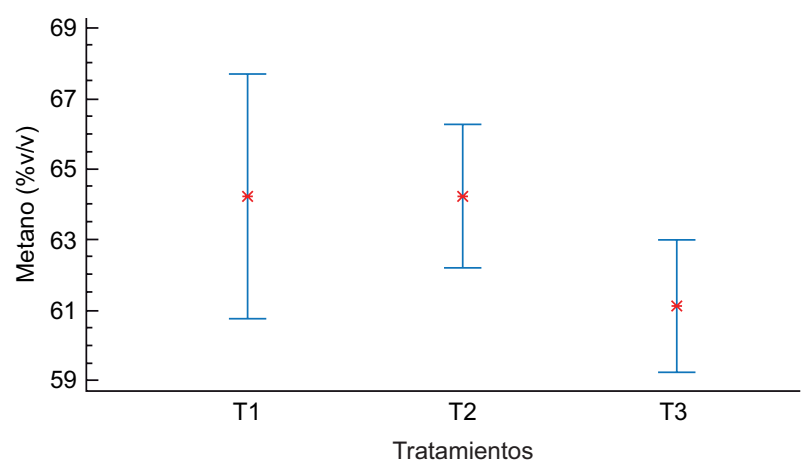

Fig. 5. Análisis de varianza del $\mathrm{CH}_{4} \pm$ error estándar. $\mathrm{T} 1=$ Excretas de borrego-lodos, T2 = Excretas de borrego-rumen, $\mathrm{T} 3=$ Excretas de borrego

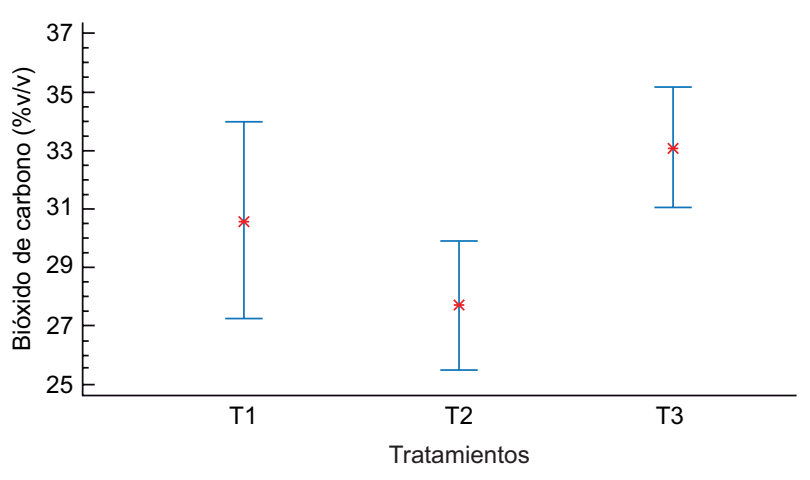

Fig. 6. Análisis de varianza del $\mathrm{CO}_{2} \pm$ error estándar. $\mathrm{T} 1=$ Excretas de borrego-lodos, T2 = Excretas de borrego-rumen, $\mathrm{T} 3=$ Excretas de borrego

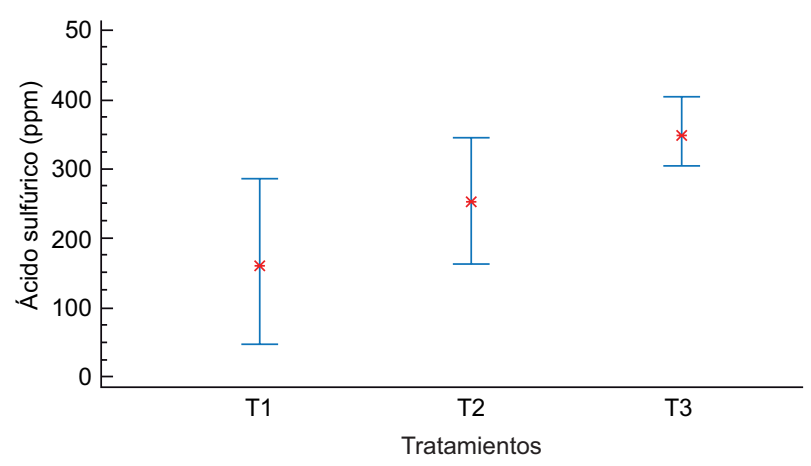

Fig. 7. Análisis de varianza del $\mathrm{H}_{2} \mathrm{~S} \pm$ error estándar. $\mathrm{T} 1=$ Excretas de borrego-lodos, T2 = Excretas de borrego-rumen, $\mathrm{T} 3=$ Excretas de borrego

El valor más alto de ácido sulfhídrico se encontró en el T3 (excretas de borrego). La prueba múltiple de Tukey mostró diferencias estadísticas significativas entre los tres tratamientos evaluados en cuanto a la producción de ácido sulfhídrico, con dicha prueba se obtuvo que el T1 y T2 son diferentes al T3.

\section{CONCLUSIONES}

El Tratamiento 1 (excretas de borrego/lodos) es el que genera menor cantidad de biogás, $35.47 \mathrm{~L}$, 
comparado con los tratamientos T 2 (excretas de borrego/rumen) y T3 (excretas de borrego), produciendo $42.14 \mathrm{~L}$ y $43.83 \mathrm{~L}$, respectivamente. La composición del biogás en cuanto a $\mathrm{CH}_{4} \mathrm{y} \mathrm{CO}_{2}$ es muy similar en los tres tratamientos. Sin embargo, el T3 presenta menor porcentaje de $\mathrm{CH}_{4}(61 \%$ contra $64 \%$ de los otros tratamientos). El contenido de $\mathrm{CH}_{4}$ en el biogás es mayor a lo calculado teóricamente, así mismo el contenido de $\mathrm{CO}_{2}$ es menor. Por otro lado, el T1 es donde se obtuvo la menor concentración de $\mathrm{H}_{2} \mathrm{~S}$. El T1 se considera el mejor, ya que presentó menor concentración de $\mathrm{H}_{2} \mathrm{~S}$. Además, que es el tratamiento que comienza a generar biogás en el menor tiempo, en comparación con los otros dos. En la práctica esto implica una reducción en el tiempo de retención de los residuos y por ende un volumen de biodigestor menor, disminuyendo costos tanto para su construcción como por el tratamiento del biogás para la eliminación del $\mathrm{H}_{2} \mathrm{~S}$.

\section{AGRADECIMIENTOS}

A la Universidad Juárez Autónoma de Tabasco por el apoyo otorgado al proyecto: Biometanización de residuos orgánicos generados en Tabasco a través de la codigestión anaerobia. Financiado por el Programa de Fomento a la Investigación (PFI), Fondo 1227.

\section{REFERENCIAS}

Álvarez R. y Lidén G. (2009). Low temperature anaerobic digestion of mixtures of llama, cow and sheep manure for improved methane production. Biomass Bioenerg. 33, 527-533. DOI:10.1016/j.biombioe.2008.08.012

Ashekuzzaman S. y Tjalfe G. (2011). Optimizing feed composition for improved methane yield during anaerobic digestion of cow manure based waste mixtures. Bioresource Technol. 102, 2213-2218.

DOI: 10.1016/j.biortech.2010.09.118

Borowski S., Dománski J. y Weatherley L. (2013). Anaerobic co-digestion of swine and poultry manure with municipal sewage sludge. Waste Manage. 34, 513-521. DOI: 10.1016/j.wasman.2013.10.022

Borowski S. y Weatherley L. (2013). Co-digestion of solid poultry manure with municipal sewage sludge. Bioresource Technol. 142, 345-352. DOI: 10.1016/j.biortech.2013.05.047

Bux R., Razaque A. y Aslam M. (2012). Biomethanization potential of waste agricultural Biomass in Pakistán: a case study. Biomass and Renewables 1, 32-37.
Flotats X., Campos E., Palatsi J. y Bonmatí X. (2001). Digestión anaerobia de purines de cerdo y codigestión con residuos de la industria alimentaria. Porci; Monografías de actualidad. 65, 51-65.

Karki A., Shrestha J. y Bajgain S. (2005). Biogas: As renewable source of energy in Nepal: Theory and development. BSP-Nepal. Kathmandu, Nepal, 189 pp.

Lay J., Li Y. y Noike T. (1997). Influences of $\mathrm{pH}$ and moisture content on the methane production in high solids sludge digestion. Water Res. Water Research 31, 1518-1524. DOI: 10.1016/S0043-1354(96)00413-7

LeJeune J. y Wetzel A. (2007). Preharvest control of Escherichia coli O157 in cattle. J. Anim. Sci. 85, E73-E80. DOI: $10.2527 /$ jas.2006-612

Miner J., Humenik F. y Overchash M. (2000). Managing livestock wastes to preserve environmental quality. Environmental Quality. Iowa State Univertisy Press. Ames, IA, EUA, 318 pp.

SE (1984a). Norma Mexicana NMX-AA-16-1984. Protección al ambiente - Contaminación del suelo - Residuos sólidos municipales - determinación de humedad. Secretaría de Economía. Dirección General de Normas. 14 de diciembre de 1984.

SE (1984b). Norma Mexicana NMX-AA-18-1984. Protección al ambiente - Contaminación del suelo - Residuos sólidos - determinación de cenizas. Secretaría de Economía. Dirección General de Normas. 10 de diciembre de 1984.

SE (1985). Norma Mexicana NMX-AA-15-1985. Protección al ambiente - Contaminación del suelo - residuos sólidos municipales - muestreo - método de cuarteo. Secretaría de Economía. Dirección General de Normas. 18 de marzo de 1985.

SE (2001). Norma Mexicana NMX-AA-034-SCFI-2001. Análisis de agua - determinación de sólidos y sales Disueltas en aguas naturales, residuales y Residuales tratadas - método de prueba. Secretaría de Economía. Dirección General de Normas. 1 de agosto de 2001.

Sharpe R. y Skakkebæk N. (1993). Are oestrogens involved in falling sperm counts and disorders of the male reproductive tract?. The Lancet 341, 1392-1395. DOI: 10.1016/0140-6736(93)90953-E

SIAP (2012a). Resumen nacional población ganadera, avícola y apícola. Servicio de Información Agroalimentaria y Pesca. Secretaría de Agricultura, Ganadería, Desarrollo Rural, Pesca y Alimentación. Base de datos. México, D.F. Consultado 31 de mayo del 2016. [en línea]. http://www.campomexicano.gob.mx/ portal_siap/Integracion/EstadisticaBasica/Pecuario/ PoblacionGanadera/Resumen/Resumen.pdf.

SIAP (2012b). Población ganadera: Ovino. Servicio de Información Agroalimentaria y Pesca. Secretaría de Agricultura, Ganadería, Desarrollo Rural, Pesca y 
Alimentación. Base de datos. México, D.F. Consultado 31 de mayo del 2016. [en línea] http://www. campomexicano.gob.mx/portal_siap/Integracion/ EstadisticaBasica/Pecuario/PoblacionGanadera/ProductoEspecie/ovino.pdf.
Weiland P. (2010). Biogas production: Current state and perspectives. Appl. Microbiol. Biotechnol. 85, 849860. DOI: $10.1007 / \mathrm{s} 00253-009-2246-7$ 